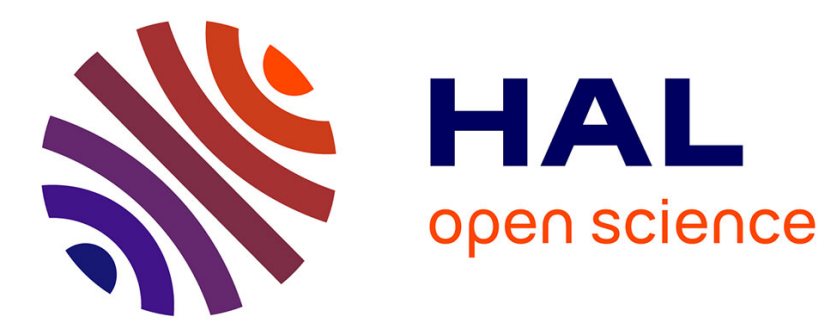

\title{
Reflective Spin-Orbit Geometric Phase from Chiral Anisotropic Optical Media
}

\author{
Mushegh Rafayelyan, Georgiy Tkachenko, Etienne Brasselet
}

\section{To cite this version:}

Mushegh Rafayelyan, Georgiy Tkachenko, Etienne Brasselet. Reflective Spin-Orbit Geometric Phase from Chiral Anisotropic Optical Media. Physical Review Letters, 2016, 116 (25), pp.253902 (1-5). 10.1103/PhysRevLett.116.253902 . hal-01346264

\section{HAL Id: hal-01346264 https://hal.science/hal-01346264}

Submitted on 18 Jul 2016

HAL is a multi-disciplinary open access archive for the deposit and dissemination of scientific research documents, whether they are published or not. The documents may come from teaching and research institutions in France or abroad, or from public or private research centers.
L'archive ouverte pluridisciplinaire HAL, est destinée au dépôt et à la diffusion de documents scientifiques de niveau recherche, publiés ou non, émanant des établissements d'enseignement et de recherche français ou étrangers, des laboratoires publics ou privés.

\section{다(1)(2)}

Distributed under a Creative Commons Attribution - ShareAlikel 4.0 International 


\title{
Reflective Spin-Orbit Geometric Phase from Chiral Anisotropic Optical Media
}

\author{
Mushegh Rafayelyan, Georgiy Tkachenko, and Etienne Brasselet* \\ Université Bordeaux, LOMA, UMR 5798, F 33400 Talence, France \\ CNRS, LOMA, UMR 5798, F 33400 Talence, France
}

\begin{abstract}
We report on highly reflective spin orbit geometric phase optical elements based on a helicity preserving circular Bragg reflection phenomenon. First, we present a dynamical geometric phase experiment using a flat chiral Bragg mirror. Then, we show that shaping such a geometric phase allows the efficient spin orbit tailoring of light fields without the need to fulfill any condition on birefringent phase retardation, in contrast to the case of transmission spin orbit optical elements. This is illustrated by optical vortex generation from chiral liquid crystal droplets in the Bragg regime that unveils spin orbit consequences of the droplet's curvature. Our results thus introduce a novel class of geometric phase elements "Bragg Berry" optical elements.
\end{abstract}

Spin-orbit interaction of light refers to the interplay between the polarizational (spin) and spatial (orbital) degrees of freedom of light. As recently reviewed in [1], a general framework based on optical angular momenta and geometric phases allows one to unravel the various manifestations of spin-orbit interaction of light. Since geometric phases are associated with rotations of the coordinate system and lead to helicity-dependent phases for circularly polarized light [2,3], they offer a robust road map towards the elaboration of spin-controlled optical elements. In particular, space-variant optically anisotropic planar structures have attracted a lot of interest for more than a decade. In the transmission mode, such structures give a maximal spin-orbit effect when the half-wave retardation condition is fulfilled [4,5]. More recently, nanofabrication technologies have led to the realization of metasurfaces - planar optical elements with subwavelength thickness - that allow the efficient shaping of the amplitude, phase, and polarization state of light in an arbitrary manner, as reviewed in [6,7].

In this context, here we propose an original spin-orbit approach to tailor optical fields via the geometric phase acquired by light as it reflects from chiral anisotropic media. Our idea relies on the use of chiral nematic liquid crystals-cholesterics [8] - that combine an intrinsic periodic modulation of the molecular orientation (described by a unit vector $\mathbf{n}$ called director, $\mathbf{n}$ and $-\mathbf{n}$ being equivalent) with chiral supramolecular ordering characterized by pitch $p$ (that is the distance over which the director rotates by $2 \pi$ ). In turn, a circularly polarized wave satisfying the Bragg condition $\Lambda \chi=-1(\Lambda= \pm 1$ being the helicity of light and $\chi= \pm 1$ referring to the right or left handedness of the supramolecular chirality) may not propagate for a range of frequencies that depends on the refractive indices of the cholesteric material. Practically, this corresponds to almost a $100 \%$ reflection of light for a thick enough sample, which is the manifestation of the circular Bragg-reflection phenomenon $[9,10]$. This is illustrated in Fig. 1(a) that emphasizes an important feature of circular Bragg reflection-the preservation of helicity-which implies flipping of the projection of the photon spin angular momentum along the propagation axis $z$.

Let us now set a planar cholesteric film into rotation and reveal a dynamic geometric phase associated with such a circular Bragg mirror. For this purpose, the twodimensional (2D) mirror is spun around its normal axis $z$ (with the unit vector $\mathbf{z}$ ) at an angular frequency $\Omega=\Omega \mathbf{z}$ and illuminated by a laser beam of wavelength $\lambda_{0}=$ $532 \mathrm{~nm}$ that falls into the Bragg-reflection spectral range under the condition $\Lambda \chi=-1$, see Fig. 1(b). As a result, the cholesteric helices rotate around the $z$ axis at an angular frequency $\Omega$, as sketched in Fig. 1(c). In the rotating coordinate system "attached" to the director field, the light acquired the geometric phase $\Phi=-\int \mathbf{s} \cdot \Omega d t$ [12], where $\mathbf{s}$ is the spin angular momentum (per photon, in $\hbar$ units) aligned along the beam propagation direction axis, and $t$ is the time. Since the Bragg field satisfies $\mathbf{s}^{(i)} \cdot \Omega=\chi \Omega$ and $\mathbf{s}^{(r)} \cdot \Omega=-\chi \Omega$, we have $\Delta \Phi=\Phi^{(r)}-\Phi^{(i)}=-2 \chi \Omega t$. In other words, the Bragg-reflected wave experiences an angular frequency shift $\partial(\Delta \Phi) / \partial t=-2 \chi \Omega$.

Experimentally, the above prediction is verified by recording the net optical power of the Bragg-reflected wave combined with the unavoidable contribution of Fresnel reflection at air-glass interfaces of the cell. While the Bragg part of the reflected light is expected to acquire a frequency shift $-2 \chi \Omega$, the Fresnel one is not frequency shifted since reflection off a glass surface does not flip the photon spin angular momentum, as is the case for a usual mirror. In turn, we aim at detecting the temporal beatings that result from the superposition of these two parts of the reflected light.

Noting that the Bragg and Fresnel contributions have orthogonal polarization states and different power [13], we 


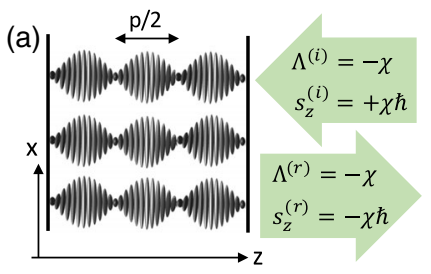

(b)

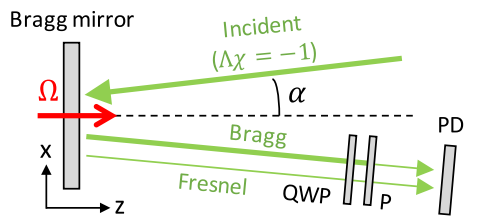

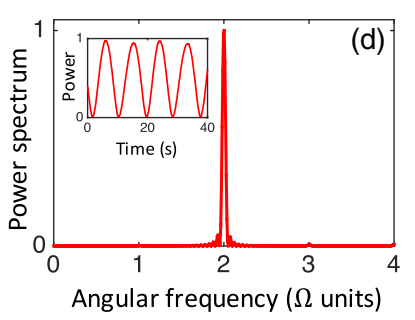

(c)

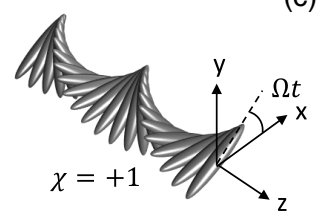

FIG. 1. (a) Side view of the supramolecular helical ordering of the chiral liquid crystal planar Bragg mirror and incident $(i)$ and reflected $(r)$ optical features associated with the circular Bragg reflection. The mirror is made of a right handed $(\chi=+1)$ cholesteric film of thickness $L=5 \mu \mathrm{m}$ and pitch $p=347 \mathrm{~nm}$ (MDA 023211 from Merck [11]). (b) Dynamical geometric phase experiment setup. The slightly oblique incidence $\left(\alpha \simeq 4^{\circ}\right)$ allows the analysis of raw reflected light. QWP, quarter wave plate; P, polarizer. The thin "Fresnel" arrow refers to incident light reflected at air glass interfaces and the thick arrow labeled "Bragg" refers to circular Bragg reflection. The sample is rotated at an angular frequency $\Omega=20^{\circ}$ s. (c) Illus tration of the rotation of the supramolecular helix at an angular frequency $\Omega$. (d) Power Fourier spectrum of the periodic signal (see inset) acquired by the photodetector PD shown in panel (b) over $\simeq 40$ periods.

use a quarter-wave plate (QWP) and a linear polarizer $(\mathrm{P})$ whose relative orientation is chosen to ensure an optimum contrast of the interference pattern impinging on the photodetector, see Fig. 1(b). As shown in Fig. 1(d), the detected signal is periodic and its power Fourier spectrum reveals a peak frequency at $2 \Omega$ [14]. This matches with the picture of a linearly polarized light having a polarization plane rotating at an angular frequency $\Omega$ that passes through a linear polarizer. Practically, we used slightly oblique incidence $\left(\alpha \simeq 4^{\circ}\right)$ that allows better direct comparison with the above presentation of the dynamical geometric phase effect since raw reflected fields can be analyzed.

Next, in addition to the time-variant rotation of the supramolecular director helix, we exploit its space-variant rotation, in order to generalize the concept of dynamic geometric phase associated with chiral Bragg mirrors. The idea relies on the geometric phase universality regarding temporal or spatial rotations as discussed in [12]. As an experimental proof, here we report on the generation of optical vortices by circular Bragg reflection off cholesteric droplets.

Namely, we consider a spherical droplet having parallel surface anchoring conditions, which is obtained in practice by the mechanical stirring of cholesteric liquid crystal in glycerol or water. In the most common situation, which is
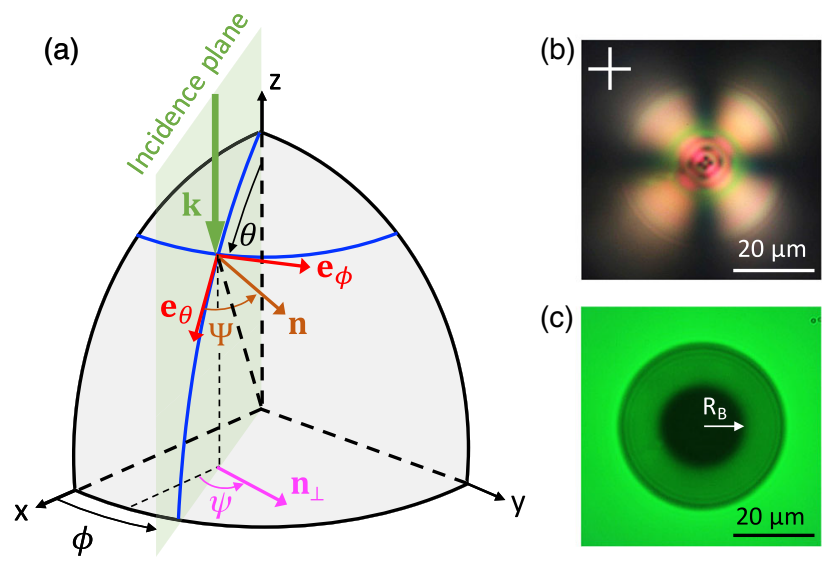

FIG. 2. (a) Definition of the parameters involved in the para metrization of the 3D director field of a radial cholesteric droplet in the spherical coordinate system $(r, \theta, \phi)$. The incidence plane and incident wave vector $\mathbf{k}$ in the case of illumination towards $z<0$ is also shown. (b) Incoherent white light imaging of a radial cholesteric droplet immersed in glycerol between crossed linear polarizers whose orientations are given by the white cross. (c) Transmission image of the droplet under circularly polarized incoherent illumination at $532 \mathrm{~nm}$.

the case in this Letter, the three-dimensional (3D) director field of such a droplet is described in the spherical coordinate system by $\left(n_{r}, n_{\theta}, n_{\phi}\right)=(0, \cos \Psi, \sin \Psi)$, where $\Psi(r, \phi)=$ $\phi+2 \pi r / p+\Psi_{0}[15]$, with $\Psi_{0}$ a constant, see Fig. 2(a). This corresponds to a radial distribution of the axis of the supramolecular helices, from which comes the name "radial cholesteric droplet." The corresponding radial symmetry is practically assessed from the observation of the droplet between crossed linear polarizers, see Fig. 2(b), while the circular Bragg reflection strikingly manifests itself from transmission imaging under Bragg circularly polarized illumination, see Fig. 2(c). Indeed, the central dark disk of radius $R_{B}$ centered on the droplet exhibited in Fig. 2(c) is reminiscent of the fact that a radial cholesteric droplet can be viewed as the curved version of the flat chiral Bragg mirror [16].

Another specificity of a radial cholesteric droplet is the presence of a radial defect line, here pointing along the direction defined by $\theta=0$, whose structure is now well understood [17]. The corresponding distribution of orientation angle $\psi$ of the director projection $\mathbf{n}_{\perp}=(\mathbf{n} \cdot \mathbf{x}) \mathbf{x}+$ $(\mathbf{n} \cdot \mathbf{y}) \mathbf{y}$ on a plane perpendicular to $z$ when looking at the droplet from the top and bottom [see Fig. 2(a) in the former case] is shown in Figs. 3(a) and 3(b), respectively. Thus, depending on whether the incident light propagates towards $z<0$ or $z>0$, the surface director structuring "viewed" by the incident light corresponds to a spatial pattern with topological charge +2 or 0 , respectively. As shown hereafter, optical phase engineering of the Bragg-reflected wave must take into account the curvature of the droplet surface, which brings an intriguing subtlety regarding the topological features "experienced" by light via light-matter interaction. 

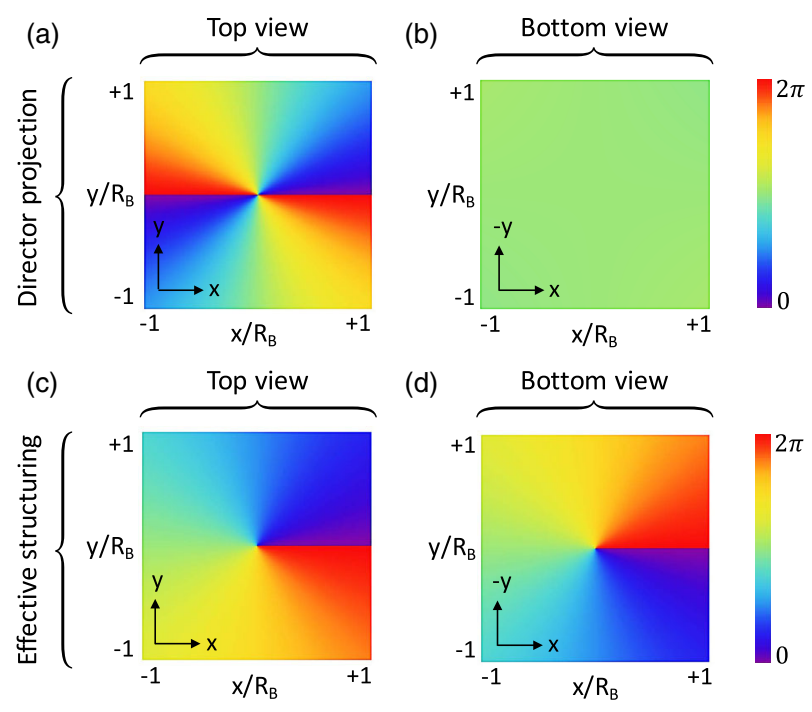

FIG. 3. (a),(b) Calculated orientation angle of the projection of the $3 \mathrm{D}$ director field at the surface of the droplet in a plane perpendicular to $z$, when looking at the droplet towards $z<0$ (top view) and $z>0$ (bottom view). (c),(d) Space variant optical axis orientation angle of an effective flat inhomogeneous chiral Bragg mirror that takes account of the curvature of the droplet surface. Without lack of generality all plots are evaluated taking $2 \pi R / p+\Psi_{0}=0$ modula $2 \pi, R$ being the droplet radius.

The curvature issue is addressed by considering an incident ray oriented towards $z<0$ impinging at the point $(R, \theta, \phi)$ as shown in Fig. 2(a). In terms of light-matter interaction, geometric phase shaping is driven by the angle $\Psi$ between the incidence plane and the director $\mathbf{n}$ in the tangent plane to the droplet. The effect of curvature can thus be described by an effective flat inhomogeneous chiral Bragg mirror with a surface space-variant director orientation angle given by $\Psi(R, \phi)=\phi+$ constant. This corresponds to a topological charge +1 , see Fig. 3(c). Correspondingly, the effective space-variant anisotropy is endowed with a topological charge -1 for an incident light propagating towards $z>0$, see Fig. 3(d). Ensuing optical vortex generation then follows from the formal analogy with the behavior of transmissive space-variant uniaxial slabs characterized by an in-plane distribution of the optical axis orientation of the form $\psi=q \phi$ ( $q$ half-integer). Namely, the amplitude of the generated output circularly polarized component having a reversed spin angular momentum projection along the propagation direction of light acquires a pure phase term of the form $\exp (\operatorname{im} \phi)$ with $m=2 q \Lambda^{(i)}[18,19]$. In the present case, the Braggreflected light field is therefore expected to acquire an optical phase singularity with the topological charge $m=-2 \chi$ for an incident wave propagating towards $z<0$ and $m=+2 \chi$ for incident propagation towards $z>0$.

An experimental approach to verification of the above reasoning implies the illumination of a radial cholesteric droplet along the direction of its radial defect. This is not an easy task since droplets in a solution always have random orientation. We address this issue by suspending a droplet in a levitation optical trap by means of the optical setup sketched in Fig. 4(a). The "levitator" basically exploits the helicity-dependent optical radiation force exerted on Bragg cholesteric droplets [16] by a circularly polarized (according to the condition for circular Bragg reflection) upward (towards $z>0$ ) optical vortex beam with topological charge $\ell= \pm 1$. Namely, a laser beam having a complex amplitude proportional to $\exp ( \pm i \phi)$ and ensuing doughnut intensity profile. Such hollow optical intensity distribution allows us to compensate the downward gravitational force acting on the droplet by the optical force component directed along the $z$ axis, while the horizontal component of the optical force keeps the droplet in a stable balance on the beam axis, see Fig. 4(c). Figuratively speaking, the vortex beam resembles an ice-cream cone holding a scoop of ice cream (the Bragg-reflecting droplet). A side view of a steady levitated droplet is shown in Fig. 4(d). Based on the axial symmetry of the intensity profile, we expect that optical forces arising from the scattering of light by the radial defect must eventually orient it along the beam axis, which follows director parametrization given above. (a)

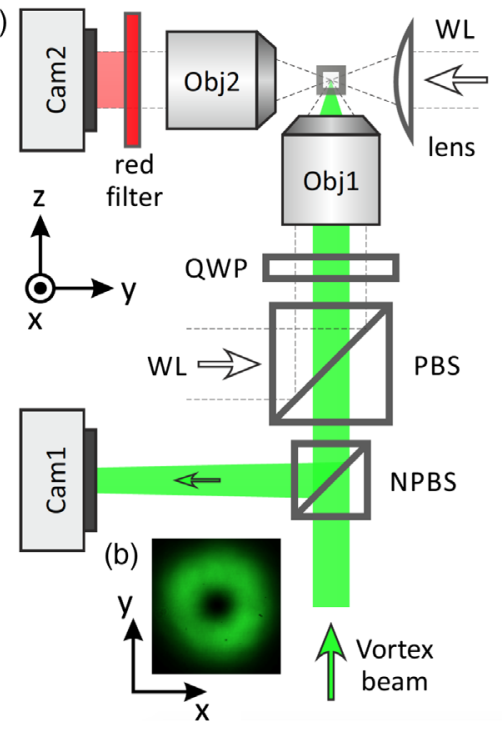

(c)
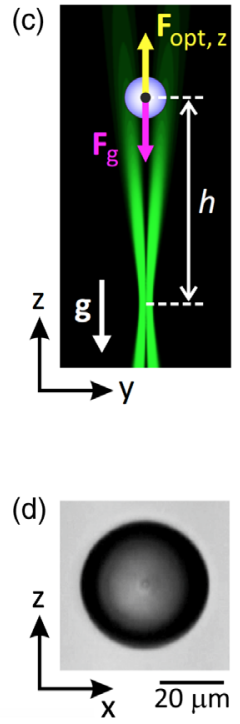

FIG. 4. Optical vortex levitation of Bragg radial cholesteric droplets. (a) Setup. QWP, quarter wave plate; (N)PBS, (non) polarizing beam splitter; Obji $i_{(i 1,2)}$, microscope objectives; Cami $i_{(i, 2)}$, CMOS cameras; WL, halogen white light illumina tion. Cholesteric droplets are prepared in milli Q water within a sealed square $\left(1 \times 1 \mathrm{~mm}^{2}\right)$ glass capillary oriented along the $x$ axis. (b) Measured transverse intensity distribution of the incident vortex laser beam $\left(\lambda_{0}=532 \mathrm{~nm}\right)$. (c) Sketch of a radial cholesteric droplet levitated by the vortex beam focused by the underfilled microscope objective Obj1 $(\times 20, \mathrm{NA}=0.4)$ and characterized by a divergence angle $\theta_{0} \simeq 6.3^{\circ}$ and beam waist radius $w_{0} \simeq 1.5 \mu \mathrm{m}$. (d) Image of a steady levitated droplet captured by Cam2. 
Indeed, this prediction is validated by our observations detailed below.

In order to analyze the geometric phase shaping produced by a spherical chiral Bragg mirror, we collected the helicity-preserved component of the reflected light by means of the illumination objective Obj1, see Fig. 4(a). After the appropriate polarization filtering ensured by QWP and polarizing beam splitter (PBS), the investigated optical field is deflected by the nonpolarizing beam splitter (NPBS) and captured by Cam1, as shown in Fig. 4(a). Prior to working with levitating droplets, we instead used the planar chiral Bragg mirror described in Fig. 1. The results of this benchmark test are shown in Figs. 5(a)-5(d). As expected, the reflected vortex beam preserved its azimuthal dependence $\exp (i \ell \phi)$ for the amplitude, as demonstrated in Figs. 5(b) and 5(d) where single-arm spiral interference patterns of opposite handedness are obtained from coherent superposition of the reflected fields shown in Figs. 5(a) and 5(c) with a copolarized collinear Gaussian beam reference.

Then, the same experiment is performed using a steady levitated droplet at fixed levitation height $h$ [see Fig. 4(c)]. The results are summarized in Figs. 5(e)-5(h). Remarkably, both the reflected intensity and phase profiles depend on $\ell$ in contrast to the 2D mirror (i.e., the slab). More precisely, the ring of the annular intensity pattern [20] is significantly larger for $\ell=+1$ than for $\ell=-1$ and the azimuthal dependence for the field amplitude is of the form $\exp [i(\ell+2) \phi]$. Indeed, the presence of optical phase singularity with topological charges +3 and -1 are, respectively, confirmed by the interference patterns in Figs. 5(f) and 5(h). These observations agree with the above assumption concerning the terminal upward orientation of the radial defect and the prediction for the Bragg-reflected field (propagating towards $z<0$ ) to gain an extra geometric phase term of the form $\exp (+i 2 \chi \phi)$, recalling that $\chi=+1$ for the chosen cholesteric material.

Concluding remarks.-Although being restricted to supra-wavelength-thick chiral samples (which noticeably brings almost $100 \%$ reflectivity), the proposed phase shaping approach is valid for a smaller thickness too. In the particular case of liquid crystals that possess a moderate refractive index, this is at the expense of reflectivity. Indeed, considering the used material with pitch $p \sim 350 \mathrm{~nm}$, a single-pitch-thick slab $(L=p)$ has a $\sim 10 \%$ reflectivity [21] and qualifies as a (non-negligibly) reflective dielectric metasurface as such $(L<\lambda)$. Interestingly, the latter reflectivity limitations can be waived with an appropriate choice of material parameters recalling that the circular Bragg photonic band gap is basically defined as $n_{\perp} p<\lambda<n_{\|} p$. One can thus envision chiral metasurfaces fabricated from high refractive index metamaterials and a small enough pitch with respect to wavelength. Indeed, this would enable the realization of a (sub)wavelength-thick slab that behaves as highly reflective geometric phase device. Importantly, Bragg-Berry chiral optical elements represent a novel class of spin-orbit components that are not required to fulfill the half-wave retardation condition for optimal vortex generation, in contrast to transmissive spin-orbit couplers based on spacevariant anisotropic slabs $[18,19]$. Moreover, the experimental identification of the topological role played by the curvature of 3D micro-optical elements emphasizes the potential of curved metasurfaces for advanced beam shaping.

Finally, we note that the present self-engineered Bragg-Berry chiral optical elements are the reflective analog of previously reported transmissive vortex generators made of nematic (nonchiral) liquid crystal droplets [22]. Since both ultimately rely on geometric phases, they are formally wavelength independent, though their efficiency remain wavelength dependent. Finally, the proposed optical
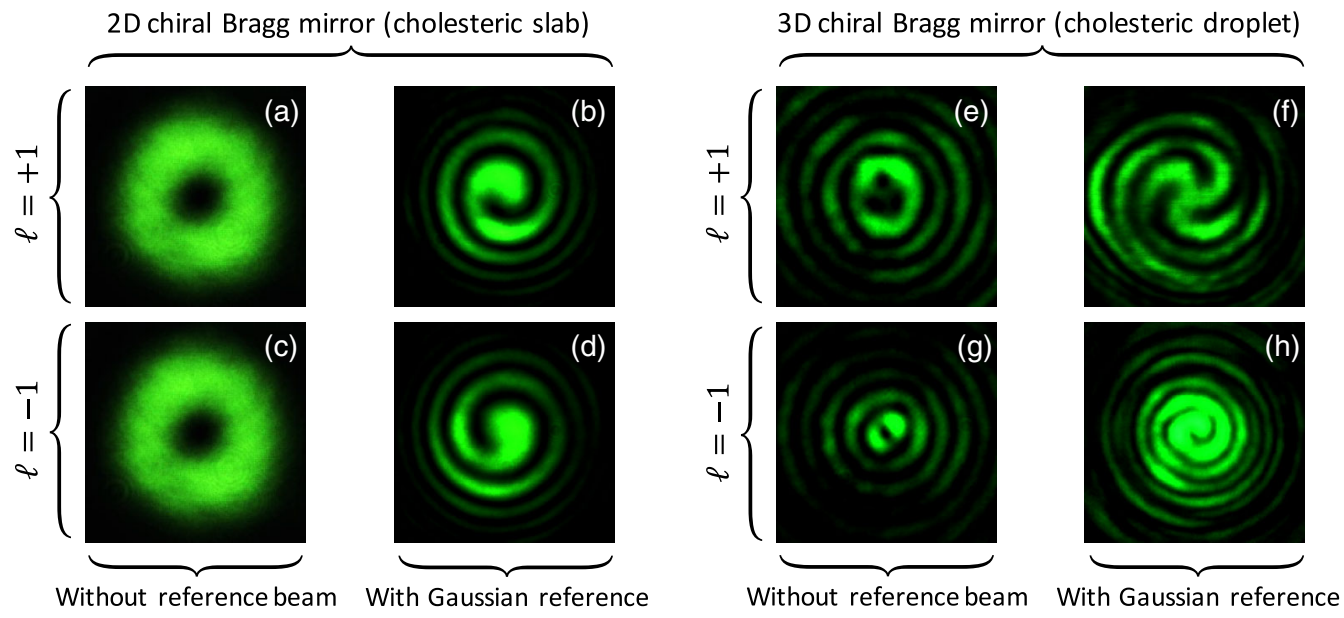

FIG. 5. (a),(b) Intensity and phase profiles retrieval for a 2D chiral Bragg mirror for the incident vortex beam with $\ell=+1$. (c), (d) Same as (a),(b) for $\ell=-1$. (e),(f) Intensity and phase profile analysis for a spherical (3D) chiral Bragg mirror (radial cholesteric droplet) for incident vortex beam with $\ell=+1$. (g),(h) Same as (e),(f) for $\ell=-1$. Experimental parameters for the 3D case: levitator beam power $P=17 \mathrm{~mW}$, levitated droplet radius $R=23 \mu \mathrm{m}$, and equilibrium elevation $h=240 \mu \mathrm{m}$. 
vortex levitation technique enriches the state-of-the-art toolbox for the manipulation of chiral micro-objects by light [23-25].

M. R. acknowledges the financial support from Erasmus Mundus Action 2 BMU-MID under Grant No. MID20121729.

Note added in the proof.-We recently became aware of the work in Ref. [26]. The authors reported on the generation of optical vortices using cholesteric liquid crystal planar slab having azimuthally patterned surface orientation of the director, discussing the role of the incidence angle and wavelength. This result provides further evidence of the interest to exploit the reflective geometric phase from chiral anisotropic media towards the elaboration of flat reflective optical elements. In addition, a similar analysis is reported in Ref. [27] where some of the authors of the present Letter demonstrate the broadband optical vortex generation using Bragg-Berry flat mirrors.

*etienne.brasselet@u bordeaux.fr

[1] K. Y. Bliokh, F. J. Rodriguez Fortuno, F. Nori, and A. V. Zayats, Spin orbit interactions of light, Nat. Photonics 9 , 796 (2015).

[2] S. I. Vinitskiŭ, V. L. Derbov, V. M. Dubovik, B. L. Markovski, and Y.P. Stepanovskiŭ, Topological phases in quantum mechanics and polarization optics, Usp. Fiz. Nauk 33, 403 (1990).

[3] R. Bhandari, Polarization of light and topological phases, Phys. Rep. 281, 1 (1997).

[4] E. Hasman, G. Biener, A. Niv, and V. Kleiner, Space variant polarization manipulation, Prog. Opt. 47, 215 (2005).

[5] L. Marrucci, E. Karimi, S. Slussarenko, B. Piccirillo, E. Santamato, E. Nagali, and F. Sciarrino, Spin to orbital con version of the angular momentum of light and its classical and quantum applications, J. Opt. 13, 064001 (2011).

[6] N. Yu and F. Capasso, Flat optics with designer metasur faces, Nat. Mater. 13, 139 (2014).

[7] G. Eleftheriades and A. Alù, Electromagnetic metasurfaces: introduction, J. Opt. Soc. Am. B 33, EM1 (2016).

[8] P. Oswald and P. Pieransky, Nematic and Cholesteric Liquid Crystals: Concepts and Physical Properties Illustrated by Experiments (Taylor \& Francis, CRC, Boca Raton, FL, 2005).

[9] D. W. Berreman and T. L. Scheffer, Bragg Reflection of Light from Single Domain Cholesteric Liquid Crystal Films, Phys. Rev. Lett. 25, 577 (1970).

[10] M. Faryad and A. Lakhtakia, The circular Bragg phenome non, Adv. Opt. Photonics 6, 225 (2014).

[11] The refractive indices parallel and perpendicular to the director are $n_{\|}=1.7013$ and $n_{\perp}=1.5064$ at $589.3 \mathrm{~nm}$ wavelength and temperature $20^{\circ} \mathrm{C}$.
[12] K. Y. Bliokh, Y. Gorodetski, V. Kleiner, and E. Hasman, Coriolis Effect in Optics: Unified Geometric Phase and Spin Hall Effect, Phys. Rev. Lett. 101, 030404 (2008).

[13] In practice, glass substrates are not antireflection coated, hence the intensity of the Fresnel contribution is a few percent of that of the Bragg contribution.

[14] Importantly, we checked that both Bragg or Fresnel con tributions taken separately do not bring a periodic signal at $2 \Omega$ with optimal modulation contrast, though residual dynamics may appear depending on the probed location of the sample, which we merely attribute to experimental imperfections of the cholesteric ordering itself, nonideal assembling of substrates, and optical adjustments.

[15] F. Xu and P. P. Crooker, Chiral nematic droplets with parallel surface anchoring, Phys. Rev. E 56, 6853 (1997).

[16] G. Tkachenko and E. Brasselet, Spin Controlled Optical Radiation Pressure, Phys. Rev. Lett. 111, 033605 (2013).

[17] D. Sec, T. Porenta, M. Ravnik, and S. Zumer, Geometrical frustration of chiral ordering in cholesteric droplets, Soft Matter 8, 11982 (2012).

[18] G. Biener, A. Niv, V. Kleiner, and E. Hasman, Formation of helical beams by use of Pancharatnam Berry phase optical elements, Opt. Lett. 27, 1875 (2002).

[19] L. Marrucci, C. Manzo, and D. Paparo, Optical Spin to Orbital Angular Momentum Conversion in Inhomogeneous Anisotropic Media, Phys. Rev. Lett. 96, 163905 (2006).

[20] The observed broken axisymmmetry is found unavoidable in practice. We attribute this issue to the imperfect flatness of capillary cells that contain the cholesteric droplets.

[21] Calculation is made using Berreman $4 \times 4$ matrix formalism [D. W. Berreman, Optics in stratified and anisotropic media: $4 \times 4$ matrix formulation, J. Opt. Soc. Am. 62, 502 (1972)].

[22] E. Brasselet, N. Murazawa, H. Misawa, and S. Juodkazis, Optical Vortices from Liquid Crystal Droplets, Phys. Rev. Lett. 103, 103903 (2009).

[23] M. Donato, J. Hernandez, A. Mazzulla, C. Provenzano, R. Saija, R. Sayed, S. Vasi, A. Magazzu, P. Pagliusi, R. Bartolino, P. Gucciardi, O. Marago, and G. Cipparrone, Polarization dependent optomechanics mediated by chiral microresonators, Nat. Commun. 5, 3656 (2014).

[24] G. Tkachenko and E. Brasselet, Optofluidic sorting of material chirality by chiral light, Nat. Commun. 5, 3577 (2014).

[25] G. Tkachenko and E. Brasselet, Helicity dependent three dimensional optical trapping of chiral microparticles, Nat. Commun. 5, 4491 (2014).

[26] J. KobashiH. Yoshida and M. Ozaki, following Letter, Polychromatic Optical Vortex Generation from Patterned Cholesteric Liquid Crystals, Phys. Rev. Lett. 116, 253903 (2016).

[27] M. Rafayelyan and E. Brasselet, Bragg Berry mirrors: reflective broadband q plates, arXiv:1606.02762v1. 